\title{
Pattern Approximation Based Generalized Image Noise Reduction Using Adaptive Feedforward Neural Network
}

\author{
Nagaraj Bhat $^{1}$, U. Eranna ${ }^{2}$, Manoj Kumar Singh ${ }^{3}$ \\ ${ }^{1}$ Department of Electronics \& Communication Engineering, R V College of engineering, India \\ ${ }^{2}$ Head of Department, Department of Electronics \& Communication Engineering, BITM, India \\ ${ }^{3}$ Director, Manuro Tech Research Pvt. Ltd., India
}

\section{Article Info}

Article history:

Received Jan 30, 2018

Revised Jul 2, 2018

Accepted Jul 22, 2018

\section{Keyword:}

Adaptive slope

Feedforward architecture

Neural Network

Noise reduction

Pattern approximation

\begin{abstract}
The problem of noise interference with the image always occurs irrespective of whatever precaution is taken. Challenging issues with noise reduction are diversity of characteristics involved with source of noise and in result; it is difficult to develop a universal solution. This paper has proposed neural network based generalize solution of noise reduction by mapping the problem as pattern approximation. Considering the statistical relationship among local region pixels in the noise free image as normal patterns, feedforward neural network is applied to acquire the knowledge available within such patterns. Adaptiveness is applied in the slope of transfer function to improve the learning process. Acquired normal patterns knowledge is utilized to reduce the level of different type of noise available within an image by recorrection of noisy patterns through pattern approximation. The proposed restoration method does not need any estimation of noise model characteristics available in the image not only that it can reduce the mixer of different types of noise efficiently. The proposed method has high processing speed along with simplicity in design. Restoration of gray scale image as well as color image has done, which has suffered from different types of noise like, Gaussian noise, salt \&peper, speckle noise and mixer of it
\end{abstract}

Copyright (c) 2018 Institute of Advanced Engineering and Science. All rights reserved.

\section{Corresponding Author:}

Nagaraj Bhat,

Manoj Kumar Singh,

Manuro Tech Research Pvt. Ltd,

\#20,2 ${ }^{\text {nd }}$ Cross, Jyothi Nagar, Vidyaranyapura, Bangalore, India.

Email: mksingh@manuroresearch.com

\section{INTRODUCTION}

Noise arises as a result of modelled or unmodellable processes happening within the production and capturing of a real signal. It is not a part of the perfect signal and is caused by a variety of sources like variation within the detector sensitivity, environmental variations, the nature of radiation, transmission or quantization errors etc. The characteristics of noise rely on its supply. Several image processing packages contain operators which add the noise artificially to a picture. Deliberately corrupting an image with noise permits us to check the resistance of a picture and assess the performance of the image by various noise filters. Noise can be classified into two categories in which the first one depends upon image knowledge and the second one is independent of image knowledge.

There are several different types of noise can appear under the category of image data dependent noise like detector noise, speckle noise, salt \& pepper noise and poission noise. Each type of noise has their own source of origin and their effect over the image are also unique. Detector noise present in nearly all recorded images and the main reason of this noise is the discrete nature of radiation. Such kind of noise can modeled as an additive model through Gaussian distribution. Speckle noise is caused by an error in the process of transmission and corrupted pixels obtained either with the maximum value or having single bits 
flipped over. Salt \& pepper noise is caused by an error in the transmission process. Corrupted pixels by this noise are obtained by the extreme value either at maximum or minimum. Poisson noise occurs because of nonlinearity available in the detector and recorder.

Restoration of image is an important and challenging problem in image processing. Practically, additive Gaussian noise and Impulse noise model can represent the various different types of noises that appear in the image. In the case of additive the Gaussian noise model,each pixel of the image gets the change through zero-mean Gaussian distribution value. Such noise can be effectively removed by local averaging process. Linear filter like Gaussian filter can remove such noise in an efficient manner but blur the edges. In impulse noise model, only a certain percentage of the total pixels present in the image get a change by random value and keeping the remaining pixels as it.

Affected pixels with such noise have very different intensity in compared to their neighbors. It is difficult to remove the noise using Gaussian filters, because sharp changes in pixel intensity are treated as edge. To remove such type of noise, In most cases, Median or rank statistics based concepts are applied to develop the filters. But such filters cannot efficiently remove the Gaussian noise. In result ,in most of the conventional methods, to remove the noise in any received image, first noise characteristics available in the image have to estimate and then filter has to be designed accordingly. This is time consuming and costly process, not only that there is no guarantee of desired success. There is very less research has been done in the design of filter which could take care of image noise irrespective of their model characteristics. The situtation is more critical when there is mixture of noise available in the image, for example, if noisy image transmitted through the faulty transmission lines.

In this paper, knowledge-based approach using artificial neural network (ANN) has applied to overcome the issues available with conventional noise restoration approaches. ANN has the capability to acquire the knowledge from inputs through learning procedure and proven to be universal approximator [1]. In this paper perceptron based multilayer feedforward architecture has applied to restore the images. The slope of activation function plays the very important role in defining the convergence quality of learning. A less slope can cause small changes in output even there is a high change in input while high value of slope will have the reverse effect.

Hence, rather than learning with predetermine fixed slopes for all activation functions, optimal value of slope for each activation function has achieved in each iteration of learning by making them self adaptive. To understand the benefit of adaptive slopes over fixed one, a function approximation problem has considered and observed that there is a significant improvement. Later, an adaptive architecture has applied to learn the correlation patterns available in normal image pixels with their surrounding pixels using gradient method. Acquired knowledge has utilized to re-correct the relationship which has been distorted because of the presence of noise. This knowledge based recorrection doesn't dependent upon the model of noise and can remove nearly all types of noises in their individual presence or in a mixed form. The optimal size of the architecture has decided with comparative performances over various architectures and observed that smaller size could be the better choice. The proposed concept of noise restoration has also applied over color images also by providing the individual training over the available primary color information.

There are a number of researchers, who has given attention towards noise reduction. In [2], image denoising has been done through the image composition. It is applied to find the components of image achieved in moving frame that defines its local spatial information.Concept of global filtering has proposed in [3] by estimating each pixel as a function of all available pixels in the image. Based on nonlocal self similarity and the low rank approximation a image denoising method has been proposed in [4]. White zeromean Gaussian noise has been removed through sparse and redundant representation in [5]. A filter is intended in [6] to enhance the Kuwahara filter to reducing the noise. The planned Gabor Kuwahara filter in [6] is economical to scale back the noise while not losing the data on the edges, before the preparation of the pictures for segmentation and alternative image processing techniques.

The performance of many nonlinear filters for noise reduction in image signals has been investigated in [7]. Acquired pictures in hyperspectral imagination square measure disturbed by additive noise that typically assumes as zero-mean white one. In fact, there's still non-white noise in hyperspectral pictures (HSIs). The 2-dimensional filtering ways and multidimensional tensor decomposition algorithms cannot take away non-white noise from HSIs directly. Therefore, a pre whitening denoising answer supported tensor decomposition is planned in [8]. There are several important benefits of denoising the magnetic resonance (MR) images in the area of health care.Based on nonlocal likelihood filter, [9] has proposed a modified nonlocal filter for noise reduction in MR images.A fusion based concept has been applied in [10] to reduce the impulse noise from images which have been captured through multi sensors. Based on an ant colony algorithm and genetic algorithm image restoration has presented in [11]. Artificial fish-swarm algorithm has applied in [12] for a medical DR image enhancement. [13] Has proposed an adaptive median filtering algorithm, to remove the impulse noises in the color images. Based on the decomposition and 
interleaving, [14] has proposed a transmission technique which helps to restore the received image which was compressed by vector quantization. In [15], wavelet based weighted median filter has been proposed to do denoised the MRI image.

The rest of the paper is organized as follows: Section 2 describes the concept of proposed pattern approximation useful for noise reduction. In section 3 the details of applied adaptive slope on the activation function presented. The section 4 presents the detail experimental results and analysis for function approximation and noise restoration for gray as well as color images. Conclusion has presented in section 5.

\section{NOISE REDUCTION IN IMAGE AS PATTERN LEARNING \& APPROXIMATION}

Technically, image can be considered as variation of light intensity that forms a group of patterns in spatial domain. These patterns could also be simpler in nature or form complex patterns depending upon varying in intensity. In the digital domain area of these patterns inherent in the form of pixel values, it's terribly tough to outline these patterns through pixels globally, however it's doable to watch and perceive the characteristics of those patterns in the local region wherever the pixel area unit extremely correlate and can be thought as a neighborhood pattern outlined by pixels in this region as shown in Fig.1 \&Fig.2. Complexity and data accessible inside the patterns not solely depend upon the position of native region, however additionally size of the native region.

A very small spatial region won't carry high informative pattern wherever as terribly massive size can carry to several less correlated information. These native patterns can be considered as some kind of functional form and the complete image is the set of such functions. If there is good diversity available in the image, in the results there are different types of local function in the corresponding function set of image. Considering the universal approximation capability of feedward neural network, it is possible to train the neural network to understand the approximate version of local patterns in a image. Preseance of any type of noise, destroy the local functional characteristics, which can be corrected by the trained network. In result, there is a reduction of noise level without pre knowledge of noise characteristics through pattern recognition concept. Local pattern generated in a blockas shown in Figure 1 and Figure 2.

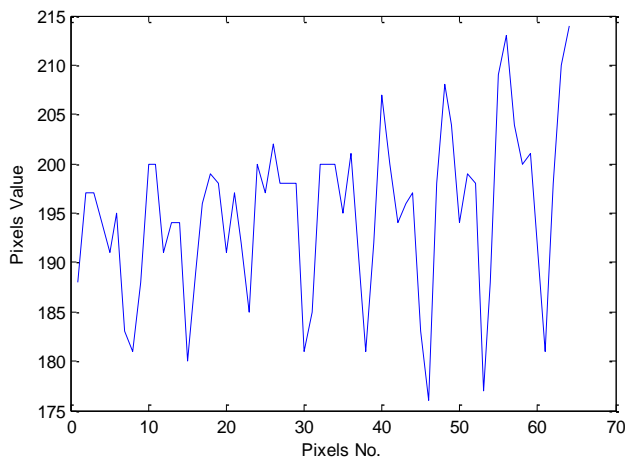

Figure 1. Local pattern generated in a block

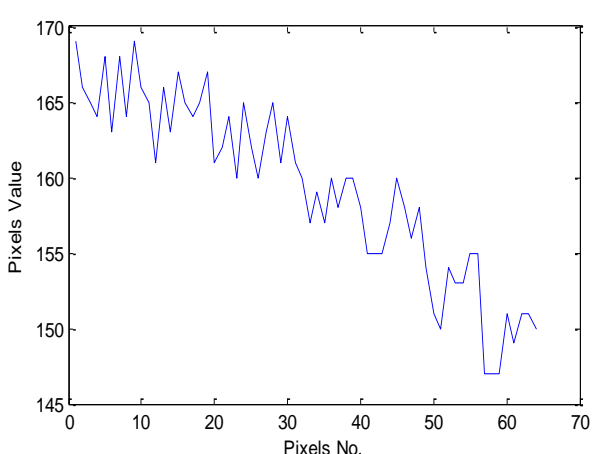

Figure 2. Local pattern generated in a block

\section{NEED FOR ADAPTIVE SLOPE ACTIVATION FUNCTION IN FF ARCHITECTURE}

The investigation of neural network approximation capabilities has been done in past by a numer of researchers. Hornik [1] has proposed the proof of universal approximation in a more generalized manner, with sufficient number of hidden units in a single hidden layer and arbitrary bounded activation function, standard multilayer feedforward networks are universal approximator with respect to $\operatorname{LP}(\mu)$ performance criteria, for arbitrary finite input environment measures $\mu$. However, he said very clearly that that all activation functions $\psi$ will not perform equally well in specific learning problems.

In the backpropagation learning process, weight updated rate is proportional to the derivative of nonlinear transfer function available with active units. In most of cases, sigmoid curve which has bell shaped derivatives has applied to MLP neural network.There may be a chance that in the training phase,output of linear accumulator may appear in the saturation region of activation function. The derivative of activation function is very small in saturation region in result learning rate becomes very slow. In result,it may take a large number of iterations to come out from this saturation region. The possible solution of this problem is to increase the region of the unsaturated region by decresing the slope of activation function. However, 
decreasing the slope will make the system closer to a linear model, which in effect diminishes the advantage of having the multilayer network. hence there is an optimum value of slope needed at each iteration as according to the landscape defined by the error function. Again the value of the slope for activation function is not same for all the neurons.

The complexity involved with MLP does not have all the slope values before training commence hence there is need to provide the adaptive mechanism which has to take care of slopes of activation function. The process for adaption of slopes can be derived simultaneously with weights optimization in terms of minimization of error function. Specifically, the slopes are to be chosen so as to minimize the performance criterion

$$
\begin{aligned}
& E_{\mathrm{q}=} \frac{1}{2}\left(\mathrm{~d}_{\mathrm{q}}-\mathrm{x}_{\text {out }}^{(\mathrm{s})}\right)^{\mathrm{T}}\left(\mathrm{d}_{\mathrm{q}}-\mathrm{x}_{\text {out }}^{\mathrm{s}}\right) \\
& =\frac{1}{2} \sum_{\mathrm{h}=1}^{\mathrm{n}}\left(\mathrm{d}_{\mathrm{qh}}-\mathrm{x}_{\text {out }, \mathrm{h}}^{\mathrm{s}}\right)^{2}
\end{aligned}
$$

Where ' $\mathrm{s}$ ' denotes the number of layers in the network and $\mathrm{d}_{\mathrm{q}} \in \mathfrak{R}^{\mathrm{n} \times 1}$ and $\mathrm{x}_{\text {out }}^{\mathrm{s}}$ are the desired and actual outputs, respectively of the network due to qth training pattern. Consider an activation function of the sigmoid type given by (2).

$$
\mathrm{f}(\mathrm{u}, \mathrm{k})=\frac{1}{\left(1+\mathrm{e}^{-\mathrm{ku}}\right)}
$$

Where $\mathrm{u}$ is the input to the nonlinearlity and $\mathrm{k}$ is the slope parameter which has to be adjusted so that (1) has to minimized. Considering the nonlinearlity of the ith neuron in the $\mathrm{s}^{\text {th }}$ layer of the network, gradient approach can be applied by obtaining

$$
\mathrm{k}_{\mathrm{i}}^{\mathrm{s}}(\mathrm{t}+1)=\mathrm{k}_{\mathrm{i}}^{\mathrm{s}}(\mathrm{t})-\beta \frac{\partial \mathrm{E}_{\mathrm{q}}}{\partial \mathrm{k}_{\mathrm{i}}^{\mathrm{s}}}
$$

Using the chain rule, the second term on the right side in (3) can be rewritten as

$$
\begin{aligned}
& \frac{\partial \mathrm{E}_{\mathrm{q}}}{\partial \mathrm{k}_{\mathrm{i}}^{\mathrm{s}}}=\frac{\partial \mathrm{E}_{\mathrm{q}}}{\partial \mathrm{u}_{\mathrm{i}}^{\mathrm{s}}} \frac{\partial \mathrm{u}_{\mathrm{i}}^{\mathrm{s}}}{\partial \mathrm{x}_{\text {out, } \mathrm{i}}^{\mathrm{s}}} \frac{\partial \mathrm{x}_{\mathrm{out}, \mathrm{i}}^{\mathrm{s}}}{\partial \mathrm{k}_{\mathrm{i}}^{\mathrm{s}}} \\
& =-\delta_{\mathrm{i}}^{\mathrm{s}} \frac{1}{\partial \mathrm{x}_{\mathrm{out}, \mathrm{i}}^{\mathrm{s}} / \partial \mathrm{u}_{\mathrm{i}}^{\mathrm{s}}} \frac{\partial \mathrm{x}_{\text {out, }}}{\partial \mathrm{k}_{\mathrm{i}}^{\mathrm{s}}}=-\delta_{\mathrm{i}}^{\mathrm{s}} \frac{\mathrm{f}_{\mathrm{k}}(\mathrm{u}, \mathrm{k})}{\mathrm{f}_{\mathrm{u}}(\mathrm{u}, \mathrm{k})}
\end{aligned}
$$

Where $\delta_{\mathrm{i}}^{\mathrm{s}}$ is the local error for the ith neuron of the sth layer, and $\mathrm{f}_{\mathrm{k}}(\mathrm{u}, \mathrm{k})$ and $\mathrm{f}_{\mathrm{u}}(\mathrm{u}, \mathrm{k})$ denote the partial derivatives of the activation function with $\mathrm{k}$ and $\mathrm{u}$ respectively. Hence the slope of the activation function can be defined by

$$
\mathrm{k}_{\mathrm{i}}^{\mathrm{s}}(\mathrm{t}+1)=\mathrm{k}_{\mathrm{i}}^{\mathrm{s}}(\mathrm{t})
$$

To increase the stability, momentum term is also added.

Learning algorithm with adaptive activation function slopes

- Initialize the weights in the network according to standard initialization process

- From set the set of training data, derive the network response

- Local error is obtained with respect to the desired network response and obtained actual output according to following equations for ouput layer and hidden layer units

for output layer:

$\delta_{\mathrm{i}}^{\mathrm{s}}=\left(\mathrm{d}_{\mathrm{q}}-\mathrm{x}_{\mathrm{out}, \mathrm{i}}^{\mathrm{s}}\right) \mathrm{g}\left(\mathrm{u}_{\mathrm{i}}^{\mathrm{s}}\right)$

for hidden layer:

$$
\delta_{\mathrm{i}}^{\mathrm{s}}=\sum_{\mathrm{h}=1}^{\mathrm{n} 2} \delta_{\mathrm{h}}^{\mathrm{s}+1} \mathrm{w}_{\mathrm{hi}}^{\mathrm{s}+1} \mathrm{~g}\left(\mathrm{u}_{\mathrm{i}}^{\mathrm{s}}\right)
$$


- The weights of the network are updated according to

$$
w_{i j}^{s}(t+1)=w_{i j}^{s}(t)+\mu \delta_{i}^{s} x_{o u t, j}^{s}
$$

- The slope of the activation function are updated according to

$$
\mathrm{k}_{\mathrm{i}}^{\mathrm{s}}(\mathrm{t}+1)=\mathrm{k}_{\mathrm{i}}^{\mathrm{s}}(\mathrm{t})+\beta \delta_{\mathrm{i}}^{\mathrm{s}}+\alpha\left[\mathrm{k}_{\mathrm{i}}^{\mathrm{s}}(\mathrm{t})-\mathrm{k}_{\mathrm{i}}^{\mathrm{s}}(\mathrm{t}-1)\right]
$$

- $\quad$ Stop the iteration if network converged, else go back to step 2.

\section{EXPERIMENTAL RUSULTS \& ANALYSIS}

The neural network is trained with large number of local patterns available in the image to map the output similar to the input to get the knowledge of possible variation within local pattern. In the noisy image, local patterns lose the inherent functional relation and this can be corrected with trained network. Care has to be taken on the learning characteristics it should be generalization not towards memorization.

\subsection{Function Approximation}

As a test case to understand the benefit of the adaptive slope in feedforward architecture, a mathematical function as defined through (6) has considered for approximation purpose. From function, 400 samples in the range of [0 4] have generated among which odd samples have taken for training purpose, while even samples have applied in the test case. Architecture of $[1,5,1]$ has developed to approximate the function with and without adaptive slope of sigmoid function and obtained performances have shown in Figure 3. The obtained value of MSE has also shown in Table 1. It is clear that there is a much better learning has happened with the adaptive slope in comparison to fixed slope of the sigmoid function. The final obtained value of the slope for hidden layer and output layer nodes has also shown in Table 2. It can also observe that such kind of values for the slopes is not possible to define through the manual approach.

$$
y=e^{-x} \sin (\pi x)+0.3
$$

Table 1. Error in learning of function approximation

\begin{tabular}{ll}
\hline Mean square error & Mean square error \\
\hline NN (Static slope) & 0.0024 \\
NN(Adaptive slope) & 0.00026 \\
\hline
\end{tabular}

Table 2. The final slope value of hidden and output node active function

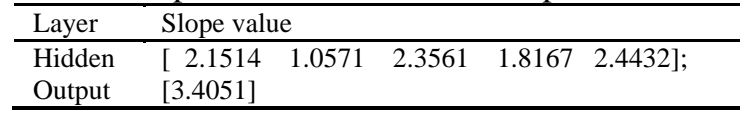

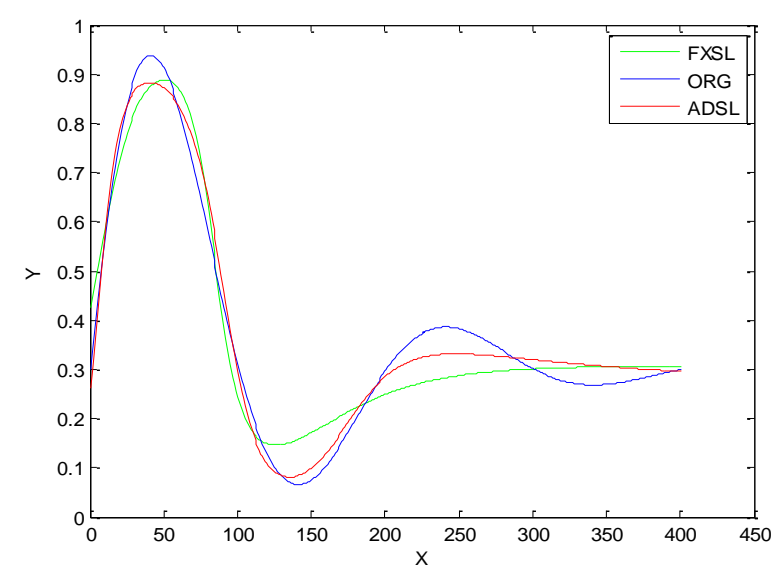

Figure 3. Function approximation by neural network 


\subsection{Image Denoising}

Quality of learning is heavily depending upon the size of the architecture and the input data. The larger size of the architecture may cause easier learning while loose the generalization capability. Hence it is very necessary to have compact size of architecture as much as possible. There is no direct way to find out the optimal size of architecture, generally various different size architecture has tested directly or indirectly like pruning method.

\subsubsection{Optimal Size of the Architecture}

To collect the optimal type of local image pattern, it is necessary that reference image must carry diverse features. Hence a gray scale of "Lena" image, having $512 * 512$ pixels has taken. Local patterns have generated through the local region pixels. To understand the optimal architecture in noise reduction different possibilities have explored as shown in Table 3. It is observed that architecture size of [ [ $\left.\begin{array}{lll}4 & 4\end{array}\right]$ has delivered the better reduction in noise in comparison to other architecture.

Table 3. PSNR $(\mathrm{db})$ values in noise reduction as function of architecture size (Noise density: 0.05, NI: noisy image)

\begin{tabular}{|c|c|c|c|c|}
\hline $\begin{array}{l}\text { Arch } \\
{\left[\begin{array}{ll}\mathrm{I} & \mathrm{H}\end{array}\right.}\end{array}$ & $\begin{array}{l}\text { litecture } \\
\mathrm{H} \quad \mathrm{O}]\end{array}$ & $\begin{array}{l}\text { Salt \&Pepper } \\
\text { [NI:18.45 ] }\end{array}$ & $\begin{array}{c}\text { Gaussian } \\
\text { [NI:19.08 ] }\end{array}$ & $\begin{array}{l}\text { Speckle } \\
\text { [NI:18.85 ] }\end{array}$ \\
\hline$[64$ & $1664]$ & 23.7797 & 22.1179 & 24.2638 \\
\hline$[64$ & 8 64] & 24.0798 & 22.1164 & 24.4328 \\
\hline$[16$ & 16] & 24.5017 & 22.5322 & 25.0232 \\
\hline$[16$ & $\left.\begin{array}{ll}4 & 16\end{array}\right]$ & 25.4475 & 22.9049 & 25.9486 \\
\hline$[16$ & $\left.\begin{array}{ll}2 & 16\end{array}\right]$ & 26.3452 & 23.0169 & 26.7370 \\
\hline$[4$ & $\left.\begin{array}{ll}2 & 4\end{array}\right]$ & 25.4106 & 22.7039 & 25.9654 \\
\hline$[4$ & 4] & 26.4235 & 23.0286 & 26.9519 \\
\hline
\end{tabular}

\subsubsection{Optimal Training Image}

The next important issue is to determine what could be nature of training image: Normal image (without noise) or Noisy image. In normal image learning same output has considered for targets, while noisy image learning as an input, corresponding normal part has considered for targets. Three versions of noisy image have developed: image corrupted by salt \& pepper noise, Gaussian noise and mixture of both. With the architecture of [ [ $\left.\begin{array}{lll}4 & 1\end{array}\right]$ for all the four different possible training images, learning has applied independently and obtained performance in noise reduction over same noise density and different noise density have shown in Table 4 and in Table 5. It is observed that normal image as a reference image for leaning has delivered the better performances in noise reduction. Hence, architecture [ $\left.\begin{array}{lll}4 & 1 & 4\end{array}\right]$ and noise free image of "Lena" has considered for final learning purpose. The convergence characteristic of learning for "Lena" image has shown in Figure 4. It is observed that there is very fast convergence has taken place. After completion of learning; different types of images with different types of noises like Gaussian noise, Salt \& Pepper noise, Speckle noise and mixtures of them have applied for test cases.

Table 4. PSNR (db) with different forms of training reference image 'Lena with [4 14 4] FF architecture size and embedded noise density: 0.05 [WN: image without noise; NI (S\&P): Image with salt \& pepper noise; NI (Gauss.): image with Gaussian noise; NI (Mix.): Image with a mixuture of noises]

\begin{tabular}{|c|c|c|c|c|c|c|}
\hline Traing Image & $\begin{array}{l}\text { Salt \& } \\
\text { Pepper }\end{array}$ & Gaussian & Speckale & $\begin{array}{c}\text { Salt \& } \\
\text { Pepper } \\
+ \text { Gaussian }\end{array}$ & $\begin{array}{c}\text { Salt \& } \\
\text { Pepper } \\
+ \text { Speckale }\end{array}$ & $\begin{array}{c}\text { Salt \& } \\
\text { Pepper } \\
+ \text { Gaussian } \\
+ \text { Speckle }\end{array}$ \\
\hline $\mathrm{WN}$ & 26.4943 & 23.0480 & 26.9568 & 22.3901 & 25.3145 & 22.2023 \\
\hline NI(S\&P) & 25.1208 & 24.9200 & 25.5793 & 23.7619 & 24.1239 & 23.0650 \\
\hline NI (Gauss.) & 23.2171 & 26.5755 & 23.4400 & 24.9156 & 22.3408 & 23.6170 \\
\hline NI (Mix.) & 19.7904 & 22.9350 & 19.9451 & 22.0384 & 19.3815 & 21.1154 \\
\hline
\end{tabular}




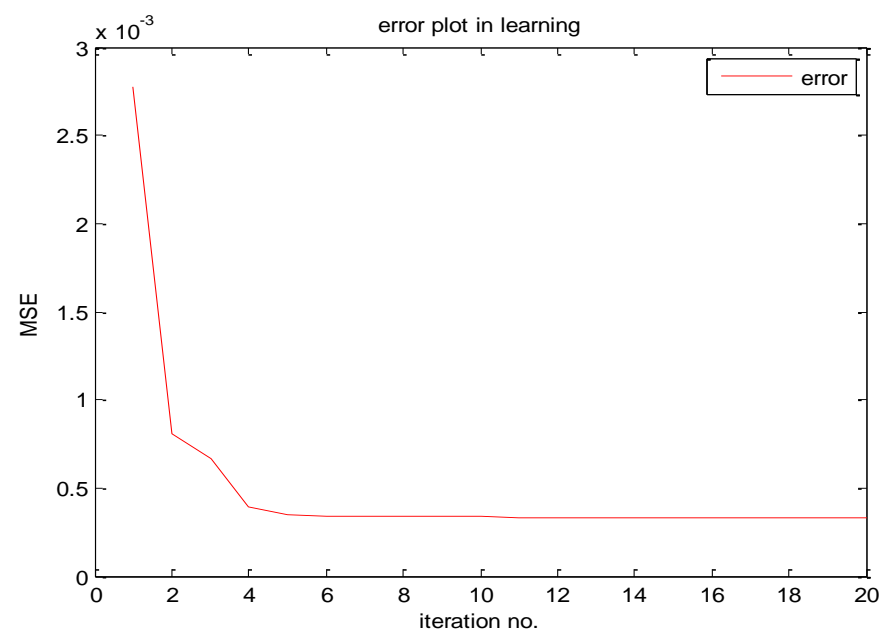

Figure 4. Learning convergences characteristic

Table 5. PSNR (db) over image with different noise density of image 'Lena with [4 1 4] FF architecture size

\begin{tabular}{llccccc}
\hline $\begin{array}{c}\text { Noise } \\
\text { density }\end{array}$ & Salt \&Pepper & Gaussian & Speckle & $\begin{array}{c}\text { Salt \& } \\
\text { Pepper } \\
\text { + Gaussian }\end{array}$ & $\begin{array}{c}\text { Salt \& } \\
\text { Pepper } \\
\text { +Speckle }\end{array}$ & $\begin{array}{c}\text { Salt \& } \\
\text { Pepper } \\
+ \text { Gaussian } \\
\text { +Speckle }\end{array}$ \\
\hline 0.1 & NI $: 15.4329$ & 17.0894 & 16.0125 & 13.8268 & 13.3473 & 12.1501 \\
& TNI : 19.2870 & 24.5692 & 19.5498 & 18.5797 & 18.4709 & 20.4951 \\
& TWN : 24.5692 & 19.0232 & 25.4949 & 23.1168 & 23.2082 & 18.9733 \\
0.01 & NI : $: 25.4161$ & 20.0374 & 25.6566 & 19.0292 & 22.7510 & 18.2995 \\
& TNI : 20.1650 & 20.6725 & 20.2324 & 20.5495 & 20.1223 & 20.4350 \\
& TWN: 28.4908 & 26.8232 & 28.6188 & 26.4790 & 28.0800 & 26.2026 \\
\hline
\end{tabular}

\subsubsection{Gray Scale Image Denoising}

Five different images have considered in this paper for the different experiment purpose as shown in the Figure 5. With each image, different types of noise: salt \& pepper noise, Gaussian noise, Speckle noise, mixture of salt \& pepper along with Gaussian and speckale noise have applied. As shown in Figure 6, different version of a noisy image in $1^{\text {st }}$ row and denoised version of the corresponding image by proposed method have shown in $2^{\text {nd }}$ row.

The corresponding PSNR values for noisy image and denoise image has shown in Table 6 and Table 7 for different noise density. Performance comparison is also presented against the Winer filter. It is observed that performance obtained with neural network is much better in comparison to the Winer filter performance. It is interesting to note that with high noise level there is more reduction in noise as observe in Table 7 in comparison to Table 6. It is also observed that mixture of noise has been handled by proposed solution very efficiently. In Figure 7, three different patterns formed by same location local pixels from noise free image (TR), noisy image (WN) and restored image (AP) has shown. It can observe that corrected pattern is very close to the true pattern.
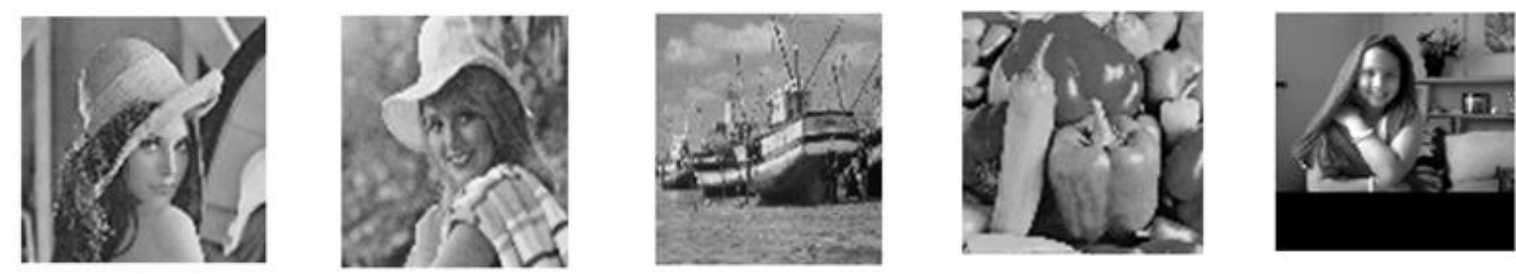

Figure 5. Images considered for experiments (from left to right): Lena, Eliane, Boat, Vegetable, and Siyasha 

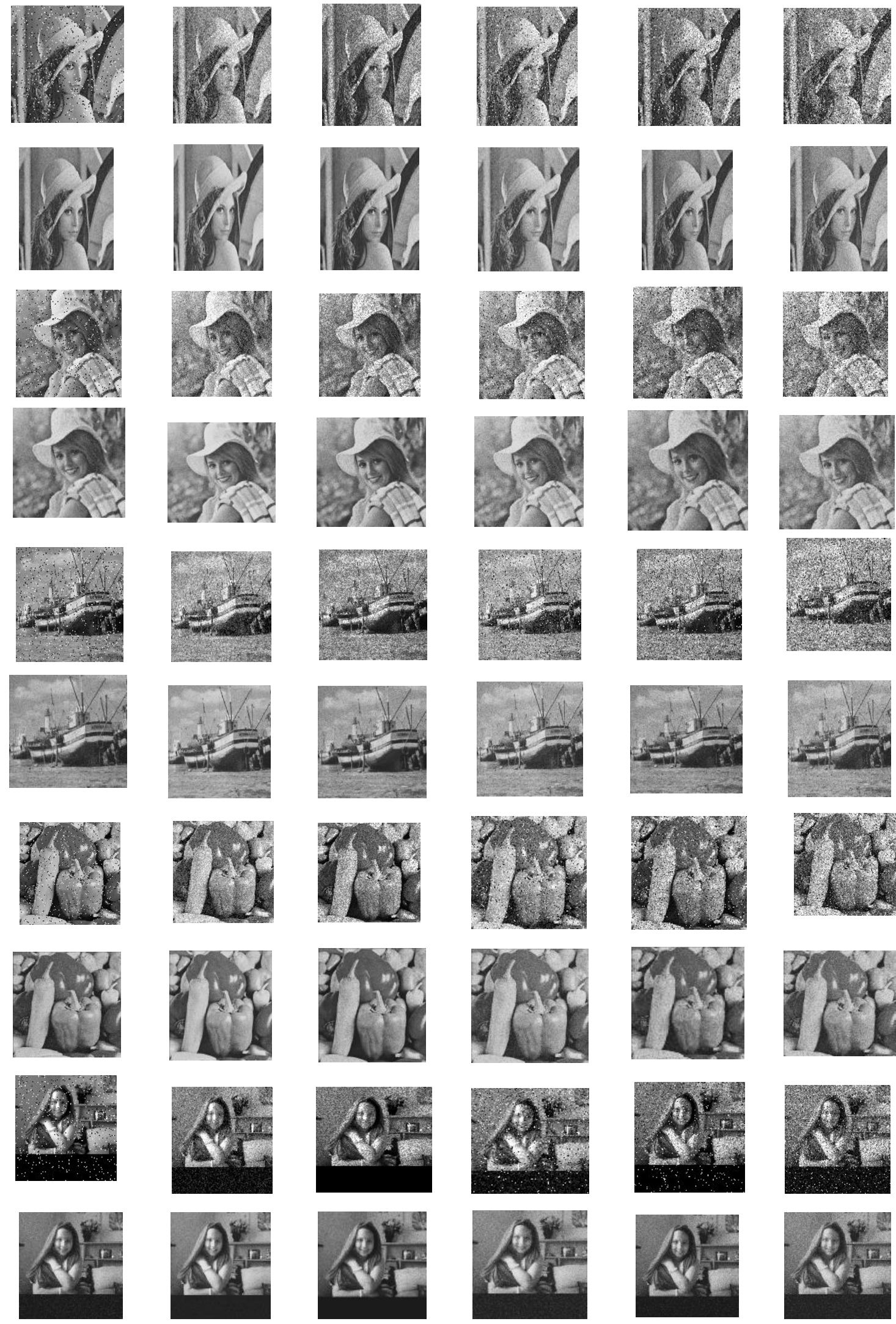

Figure 6. Denoised image from different types of noisy images (from left to right: salt\&pepper, Gaussian noise, speckale noise, salt \& pepper + Gaussian noise, salt \& pepper + speckle, salt $\&$ pepper + Gaussian + speckle noise) 
Table 6. Noise reduction in images with [ $\left.\begin{array}{lll}4 & 1 & 4\end{array}\right]$ FF architecture size with noise density: 0.05 and values are PSNR in 'db' (NI: noisy image; WD: Winer denoised image; ND: Neural denoised image)

\begin{tabular}{|c|c|c|c|c|c|c|}
\hline Image & Salt \& Pepper & Gaussian & Speckle & $\begin{array}{l}\text { Salt \& Pepper } \\
+ \text { Gaussian }\end{array}$ & $\begin{array}{l}\text { Salt \& Pepper } \\
\quad+\text { Speckle }\end{array}$ & $\begin{array}{c}\text { Salt \& Pepper } \\
\text { + Gaussian } \\
\text { +Speckle }\end{array}$ \\
\hline \multirow[t]{3}{*}{ Lena } & NI : 18.4559 & 19.1167 & 18.8197 & 16.2171 & 16.0296 & 14.5277 \\
\hline & WD : 21.1215 & 23.5830 & 24.9317 & 20.2247 & 21.1003 & 20.0890 \\
\hline & ND : $\mathbf{2 6 . 4 9 4 3}$ & 23.0480 & 26.9568 & 22.3901 & 25.3145 & 22.2023 \\
\hline \multirow[t]{3}{*}{ Eliane } & NI : 18.4129 & 19.1976 & 18.3184 & 16.2682 & 15.7437 & 14.4040 \\
\hline & WD : 21.0424 & 23.5452 & 24.4872 & 20.2975 & 20.8847 & 20.0510 \\
\hline & ND : $\mathbf{2 6 . 6 7 2 1}$ & 23.3013 & 26.7455 & 22.7778 & 25.1402 & 22.3317 \\
\hline \multirow[t]{3}{*}{ Boat } & $\mathrm{NI}: 18.4681$ & 19.0978 & 18.4746 & 16.1725 & 15.8926 & 14.3148 \\
\hline & WD : 21.1412 & 23.3198 & 24.8926 & 20.1075 & 21.1007 & 19.9033 \\
\hline & ND : $\mathbf{2 4 . 4 2 0 9}$ & 21.6640 & 24.5927 & 21.2630 & 23.6369 & 21.1711 \\
\hline \multirow[t]{3}{*}{ Vegetable } & NI : 18.3666 & 19.1179 & 18.8926 & 16.0461 & 16.0128 & 14.5515 \\
\hline & WD : 20.8791 & 23.5530 & 24.7245 & 19.9186 & 20.9233 & 19.9535 \\
\hline & ND : $\mathbf{2 5 . 3 6 8 8}$ & 22.6350 & 26.0024 & 21.8521 & 24.6091 & 21.8017 \\
\hline \multirow[t]{3}{*}{ Siyasha } & NI : 17.6320 & 19.3347 & 21.2670 & 15.7019 & 16.6772 & 14.7877 \\
\hline & WD : 19.8444 & 23.2054 & 25.9953 & 18.8413 & 20.4006 & 18.9789 \\
\hline & ND : $\mathbf{2 2 . 2 6 9 7}$ & 20.5579 & 23.4088 & 19.5326 & 22.0892 & 19.4822 \\
\hline
\end{tabular}

Table 7. Noise reduction in images with [ $\left.\begin{array}{lll}4 & 1 & 4\end{array}\right]$ FF architecture size with noise density: 0.15 and values are PSNR in 'db' (NI: noisy image; WD: Winer denoised image; ND: Neural denoised image)

\begin{tabular}{|c|c|c|c|c|c|c|}
\hline $\begin{array}{l}\text { Image } \\
(0.15)\end{array}$ & Salt \& Pepper & Gaussian & Speckle & $\begin{array}{l}\text { Salt \& Pepper } \\
+ \text { Gaussian }\end{array}$ & $\begin{array}{l}\text { Salt \& Pepper } \\
\quad+\text { Speckle }\end{array}$ & $\begin{array}{c}\text { Salt \& Pepper } \\
\text { + Gaussian } \\
\text { +Speckle }\end{array}$ \\
\hline \multirow[t]{3}{*}{ Lena } & $\mathrm{NI}: 13.6564$ & 15.0619 & 14.3692 & 12.1914 & 11.7795 & 10.7035 \\
\hline & WD : 18.6147 & 16.2731 & 20.6594 & 15.1442 & 18.1406 & 15.7941 \\
\hline & ND : $\mathbf{2 3 . 0 5 1 5}$ & 16.2163 & 24.2587 & 16.0392 & 21.5848 & 16.8653 \\
\hline \multirow[t]{3}{*}{ Eliane } & NI : 13.7623 & 15.2055 & 13.8879 & 12.3494 & 11.4638 & 10.6448 \\
\hline & WD : 18.7244 & 16.3728 & 20.3583 & 15.3999 & 17.7415 & 16.0429 \\
\hline & ND : $\mathbf{2 3 . 4 6 4 7}$ & 16.4074 & 23.6702 & 16.4933 & 20.9297 & 17.2403 \\
\hline \multirow[t]{3}{*}{ Boat } & NI : 13.7302 & 15.0086 & 13.8854 & 12.2143 & 11.5200 & 10.6461 \\
\hline & WD : 18.6937 & 16.1861 & 20.5898 & 15.1595 & 18.1028 & 15.9630 \\
\hline & ND : 22.1330 & 15.8487 & 22.7884 & 15.9444 & 20.7681 & 17.0518 \\
\hline \multirow[t]{3}{*}{ Vegetable } & $\mathrm{NI}: 13.5060$ & 15.0333 & 14.5232 & 12.0662 & 11.7499 & 10.7282 \\
\hline & WD : 18.3948 & 16.2442 & 20.4955 & 14.9512 & 17.8846 & 15.6489 \\
\hline & ND : $\mathbf{2 2 . 3 4 5 8}$ & 16.2222 & 23.9157 & 15.8794 & 21.0172 & 16.6259 \\
\hline \multirow[t]{3}{*}{ Siyasha } & $\mathrm{NI}: 12.8661$ & 14.9775 & 16.6532 & 11.3017 & 12.3061 & 10.5875 \\
\hline & WD : 17.2063 & 16.1940 & 21.2986 & 13.7651 & 17.6964 & 14.3503 \\
\hline & ND $: \mathbf{1 9 . 6 7 6 1}$ & 15.5335 & 22.4762 & 14.2980 & 19.6398 & 14.8582 \\
\hline
\end{tabular}

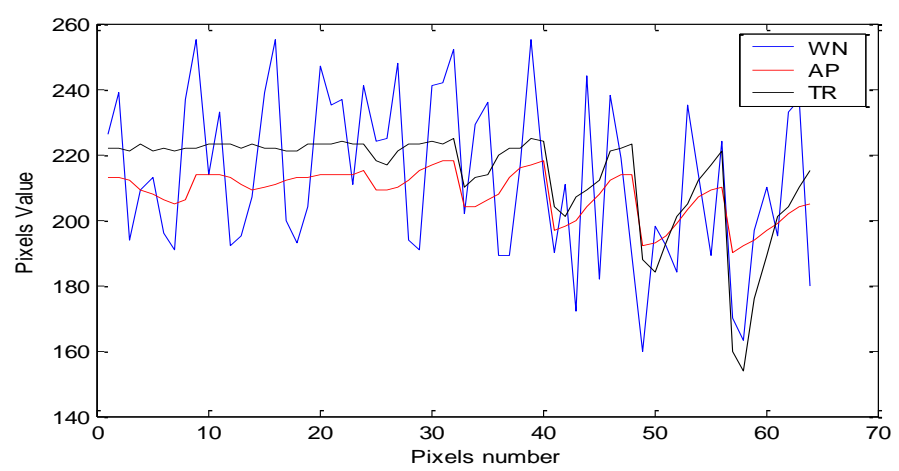

Figure 7. Recorrection of image block corrupted by speckle noise

\subsubsection{Color Image Denoise}

With the same principle as for grayscale image,color image noise restoration is also applied .Color image of Lena is taken for training purpose and individual color matrix (Red, Green \& Blue) process independently .Different types of noise have applied for test case over Lena and Vegetable Image as shown in Figure 8. The obtained performance in terms of PSNR has shown in Table 8. It is observed that there is significant improvement in noise reduction with all varieties of noise. 

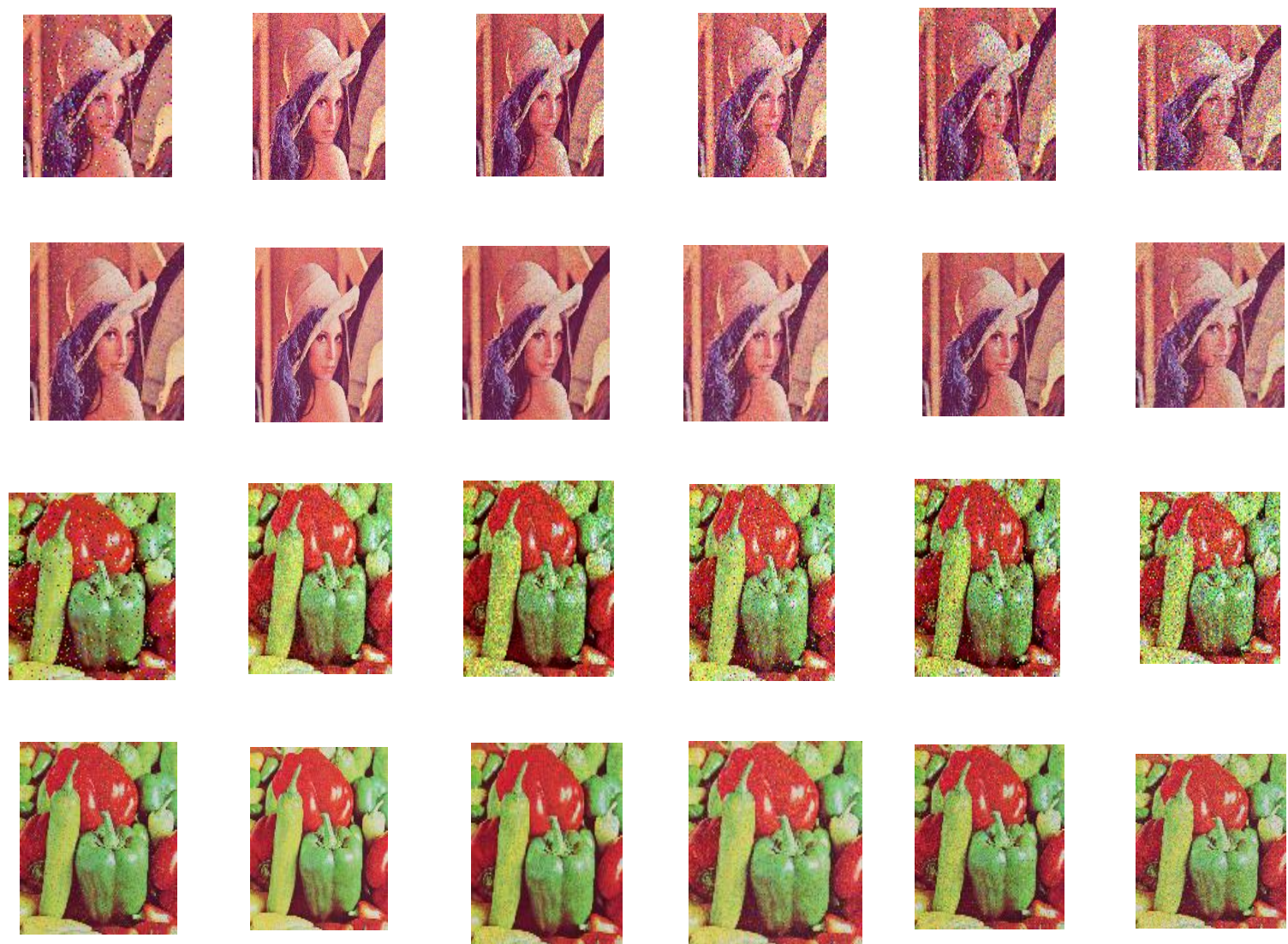

Figure 8. Denoised color image from different types of noisy images (from left to right: salt \& pepper, Gaussian noise, speckle noise, salt \& pepper + Gaussian noise, salt \& pepper + speckle, salt $\&$ pepper + Gaussian + speckle noise)

Table 8. Noise reduction in color images with [ $\left.\begin{array}{lll}4 & 1 & 4\end{array}\right]$ FF architecture size with noise density: 0.2 and values are PSNR in 'db' (NI: noisy image; ND: Neural denoised image)

\begin{tabular}{|c|c|c|c|c|c|c|}
\hline $\begin{array}{l}\text { Image } \\
(0.05)\end{array}$ & Salt \& Pepper & Gaussian & Speckale & $\begin{array}{l}\text { Salt \& Pepper } \\
+ \text { Gaussian }\end{array}$ & $\begin{array}{l}\text { Salt \& Pepper } \\
\quad+\text { Speckle }\end{array}$ & $\begin{array}{c}\text { Salt \& Pepper } \\
\text { + Gaussian } \\
\text { +Speckle }\end{array}$ \\
\hline \multirow[t]{2}{*}{ Lena } & NI $: 18.1877$ & 19.3485 & 19.1191 & 16.1515 & 15.9737 & 14.5812 \\
\hline & ND : 23.9798 & 22.5056 & 23.9612 & 20.4947 & 21.2077 & 19.4201 \\
\hline \multirow[t]{2}{*}{ Vegetable } & NI $: 17.9715$ & 19.2077 & 19.8193 & 15.9072 & 16.0824 & 14.6542 \\
\hline & ND : 21.9918 & 21.5778 & 23.1212 & 19.7357 & 20.8588 & 19.1240 \\
\hline
\end{tabular}

\section{CONCLUSION}

Universal noise restoration has proposed in this paper with intelligent manner by the use of artificial neural network. The proposed method has taken a very new concept of local pattern form by the pixels with neighboring pixels to reduce the different types of noses. If this pattern is having some kind of irregularities because of noise, approximation intelligence of neural network support enough to correct this. The proposed method performances have shown competitive performance with adaptive Weinner filter. Development of solution needed only one image at the time of training in result there is a very fast implementation of the learning process. Presented method has validated with most common types of noises in practice and the results were very satisfactory. 


\section{REFERENCES}

[1] Kurt Hornik, "Approximation Capabilities of Muitilayer Feedforward Networks", Neural Networks, Vol. 4, pp. 251-257. 1991.

[2] Gabriela Ghimpeţeanu; Thomas Batard; Marcelo Bertalmío; Stacey Levine, “A Decomposition Framework for Image Denoising Algorithms”, IEEE Transactions on Image Processing, (Volume: 25, Issue: 1, Jan. 2016).

[3] Hossein Talebi; Peyman Milanfar, "Global Image Denoising”, IEEE Transactions on Image Processing, Volume: 23, Issue: 2, Feb. 2014

[4] Qiang Guo; Caiming Zhang; Yunfeng Zhang; Hui Liu, "An Efficient SVD-Based Method for Image Denoising", IEEE Transactions on Circuits and Systems for Video Technology, (Volume: 26, Issue: 5, May 2016).

[5] Seyyed Majid Valiollahzadeh, Hamed Firouzi, Massoud Babaie-Zadeh, Christian Jutten,” Image Denoising Using Sparse Representations", (LNCS, volume 5441),ICA 2009: Independent Component Analysis and Signal Separation pp 557-564.

[6] Soumen Biswas; Dibyendu Ghoshal, "A model of noise reduction using Gabor Kuwahara filter", 2017 4th International Conference on Advanced Computing and Communication Systems (ICACCS), Year: 2017, Pages: 1 5.

[7] T. Kimura; S. Nakamura; T. Goto; S. Hirano; M. Sakurai, "Nonlinear filters for noise reduction in image signals", The 1st IEEE Global Conference on Consumer Electronics 2012 ,Year: 2012, Pages: 619 - 620

[8] Jiwei Liu; Min Fu; Xuefeng Liu, "Reduction of non-white noise from hyperspectral images based on tensor decomposition”, 2016 IEEE 13th International Conference on Signal Processing (ICSP) ,Year: 2016, Pages: 639 643.

[9] Mustapha Bouhrara; Jean-Marie Bonny; Beth G. Ashinsky; Michael C. Maring; Richard G. Spencer, "Noise Estimation and Reduction in Magnetic Resonance Imaging Using a New Multispectral Nonlocal Maximumlikelihood Filter", IEEE Transactions on Medical Imaging ,Year: 2017, Volume: 36, Issue: 1, Pages: 181 - 193.

[10] Najeeb Ullah Khan; Sajid Ullah Khan; Wang Yin Chai, "Impulse noise reduction using image fusion approach", 2016 Sixth International Conference on Innovative Computing Technology (INTECH), Year: 2016, Pages: 261 264.

[11] Yan Feng, Hua Lu and Xiliang Zeng, "Image Restoration Based on Hybrid Ant Colony algorithm", TELKOMNIKA (Telecommunication Computing Electronics and Control), Vol. 13, No. 4, December 2015, pp. 1298 1304, DOI:10.12928/TELKOMNIKA.v13i4.1900.

[12] Bi Juntao, “An Improved Medical DR Image Enhancement Method", TELKOMNIKA Indonesian Journal of Electrical Engineering, Vol. 12, No. 4, April 2014, pp. 2718 2723, DOI: http://dx.doi.org/10.11591/telkomnika.v12i4.4305.

[13] S. Abdul Saleem, T. Abdul Razak, "An Effective Noise Adaptive Median Filter for Removing High Density Impulse Noises in Color Images", International Journal of Electrical and Computer Engineering (IJECE), Vol. 6, No. 2, April 2016, pp. 611 620 .

[14] I. Elawady, A. M. Lakhdar, M. Khelifi, "Interleaved Reception Method for Restored Vector Quantization Image", TELKOMNIKA (Telecommunication Computing Electronics and Control), Vol. 14, No. 4, December 2016, pp. 1362 1367 , DOI:10.12928/TELKOMNIKA.v14i4.3181.

[15] N. Rajalakshmi, K. Narayanan, P. Amudhavalli, "Wavelet Based Weighted Median Filter for Image Denoising of MRI Brain Images", Indonesian Journal of Electrical Engineering and Computer Science (IJEECS), Vol. 10, No. 1, April 2018, pp. 201 206.

\section{BIOGRAPHIES OF AUTHORS}

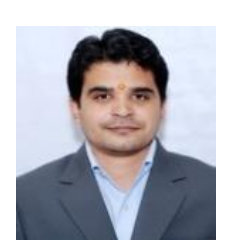

Nagaraj Bhat, received B.E degree in E\&C Engineering from VTU Belgaum in 2009 \& M.Tech in Digital Electronics from VTU in 2012. He is currently working as assistant professor in the Department of Electronics \& Communication Engineering, RV College of Engineering, Bengaluru, Karnataka, India. He is also pursuing Ph.D from VTU Belgaum. His area of interest includes Image Processing and VLSI.

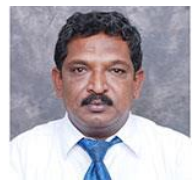

U Eranna received B.Tech degree in E\&C Engineering from JNTU Anantapur in 1988 \& M.E in Automotive Control Systems \& Robotics from M S University Baroda in 1994. He obtained Ph.D from Sri Krishnadevaraya University Anantpur in the year 2006. He is currently working as Professor \& Head of Department Electronics \& Communication Engineering BITM Bellary. His areas of Interests are communication \& Signal processing.

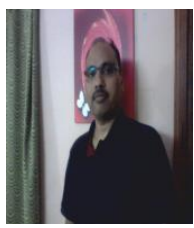

Manoj Kumar Singh is currently holding the post of Director in Manuro Tech. research Pvt.Ltd, Bangalore, India. He is also actively associated with industry and academic as expert in advanced intelligent technology. He is having background of $R \& D$ in advanced intelligent computing and solution in various fields. His field of research include Nature inspired computation, Nano-computing, Soft computing, Machine learning, Optimization, etc. 